\title{
An anti-antisigma factor in the response of the bacterium Myxococcus xanthus to blue light
}

Correspondence

Marta Fontes

mfontes@um.es

Received 19 September 2007

Revised 28 November 2007

Accepted 17 December 2007

\author{
Lilian Galbis-Martínez,† Marisa Galbis-Martínez, Francisco J. Murillo \\ and Marta Fontes
} Departamento de Genética y Microbiología (Unidad Asociada al IQFR-CSIC), Facultad de Biología,
Universidad de Murcia, 30100 Murcia, Spain

\begin{abstract}
Cells of the Gram-negative bacterium Myxococcus xanthus respond to blue light by producing carotenoids, pigments that play a protective role against the oxidative effects of light. Blue light triggers a network of regulatory actions that lead to the transcriptional activation of the structural genes for carotenoid synthesis. The product of carF, similar to a family of proteins of unknown function called Kua, is an early regulator of this process. Previous genetic data indicate that CarF participates in the light-dependent inactivation of the antisigma factor CarR. In the dark, CarR sequesters the ECF-sigma factor CarQ to the membrane, thereby preventing the activation of the structural genes for carotenoid synthesis. Using a bacterial two-hybrid system, we show here that both CarF and CarQ physically interact with CarR. These results, together with the finding that CarF is located at the membrane, support the hypothesis that CarF acts as an anti-antisigma factor. Comparison of CarF with other Kua proteins shows a remarkable conservation of a number of histidine residues. The effects on CarF function of several histidine to alanine substitutions and of the truncation of specific CarF domains are also reported here.
\end{abstract}

\section{INTRODUCTION}

Carotenoids are widely distributed, pigmented compounds that are produced by both eukaryotes and prokaryotes. These compounds protect cells from photo-oxidative damage by scavenging harmful agents, such as singlet and triplet molecular species. In the non-photosynthetic, Gram-negative bacterium Myxococcus xanthus, the synthesis of carotenoids is known to be dependent either on illumination by blue light or on exposure to copper. Much is known on the activation mechanism triggered by the light stimulus, which is brought about by a complex interplay of regulatory proteins (Elías-Arnanz et al., 2008); all but one of these proteins also participate in the carotenogenic response to copper (Fig. 1; see below).

In M. xanthus, the structural genes for carotenoid synthesis are placed at two unlinked loci. One corresponds to the single gene $c r t I$ and the other to an operon, carB, where the rest of the structural genes are grouped (Fontes et al., 1993; Botella et al., 1995). The precise mechanism for the light activation of the $c r t I$ promoter $\left(\mathrm{P}_{\mathrm{I}}\right)$ differs from that of the carB promoter $\left(\mathrm{P}_{\mathrm{B}}\right)$. However, the earlier steps that lead to

tPresent address: Servicio de Bioquímica Clínica, Hospital Gregorio Marañón, 28007 Madrid, Spain.

Abbreviation: BACTH, bacterial two-hybrid (system).

A multiple sequence alignment of the amino acid sequences of $M$. xanthus CarF and Kua proteins from different organisms is available with the online version of this paper. activation of $\mathrm{P}_{\mathrm{I}}$ as well as $\mathrm{P}_{\mathrm{B}}$ both depend on the actions of the same set of three proteins: CarQ, CarR and CarF. Two of these, a pair of sigma-antisigma factors, are the products of genes carQ and carR. They are cotranscribed, together with carS, in the carQRS operon, unlinked to other car genes and also controlled by a light-inducible promoter $\left(\mathrm{P}_{\mathrm{QRS}}\right)$. Protein CarQ, a member of the ECF family of $\sigma^{70}$ factors, is required for transcription of $\mathrm{P}_{\mathrm{I}}$ and $\mathrm{P}_{\mathrm{QRS}}$. The activity of those two promoters is very low in the dark because CarQ is sequestered by the antisigma factor CarR, a transmembrane protein. Upon illumination, CarR is somehow inactivated, and CarQ is freed from the membrane to activate its cognate promoters (Fontes et al., 1993; Hodgson, 1993; McGowan et al., 1993; Gorham et al., 1996; Martínez-Argudo et al., 1998; Browning et al., 2003). Activation of $\mathrm{P}_{\mathrm{QRS}}$ by light also requires the action of some non-specific, DNA architectural elements. One of these is IhfA, a histone-like protein, and the other is a heteromultimeric complex formed by protein CarD, bearing a DNA-binding domain that resembles eukaryotic highmobility group A proteins, and CarG, a novel zinc-binding protein (Nicolás et al., 1994, 1996; Padmanabhan et al., 2001; Moreno et al., 2001; Galbis-Martínez et al., 2004; Peñalver-Mellado et al., 2006). The $\mathrm{P}_{\mathrm{B}}$ promoter is switched off in the dark by the binding to its operator of the negative regulator CarA, produced independently of light from an adjacent operon. Light-induced activation of $\mathrm{P}_{\mathrm{B}}$ involves disrupting the CarA-operator complex (LópezRubio et al., 2004). This is achieved by CarS, the product of 


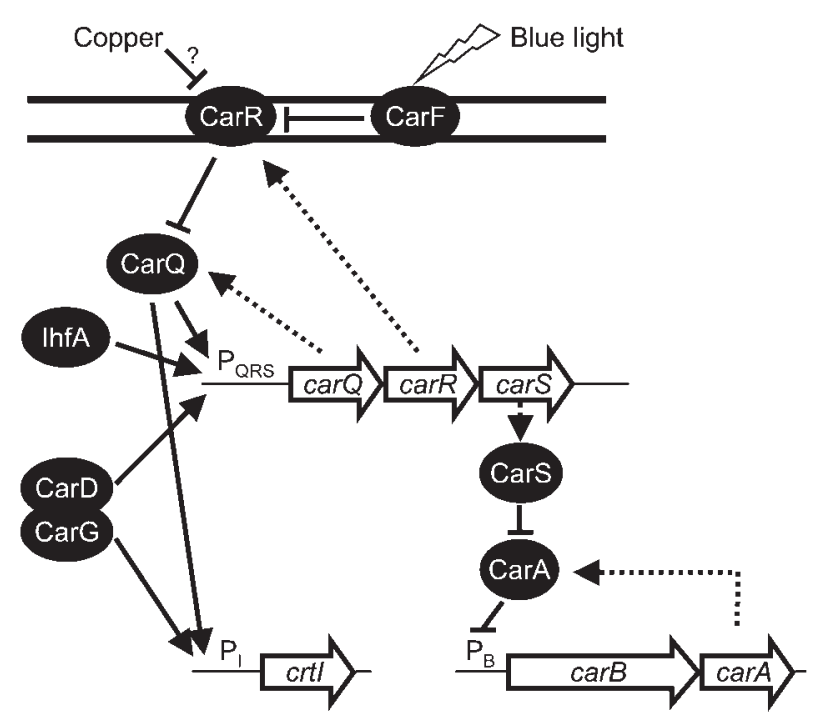

Fig. 1. The carotenogenic response of $M$. xanthus. Genes are indicated by open arrows and proteins as black ovals. Continuous arrows, positive regulation; blunt-ended lines, negative regulation. A discontinuous line joins some genes and their products. The enzymes for carotenoid synthesis are encoded in the carB operon and by the single gene crtl. Their promoters $\left(P_{B}\right.$ and $\left.P_{1}\right)$ are lightor copper-inducible. Protein CarA, constitutively expressed from the carA operon, represses $\mathrm{P}_{\mathrm{B}}$ in the dark. CarS, produced at significant levels from the carQRS operon only upon exposure to light or copper, counteracts the action of CarA. The carQRS operon also encodes CarQ, the ECF-sigma factor for its own promoter ( $\mathrm{P}_{\mathrm{ORS}}$ ) and $\mathrm{P}_{\mathrm{l}}$, and CarR, a membrane-bound antisigma factor. Also required for the activation of $P_{O R S}$ are two architectural proteins, IhfA and the CarD/CarG complex, the latter also being required to activate $P_{1}$. CarR is inactivated by exposure to light or copper. Protein CarF is absolutely necessary for the light-driven inactivation of CarR (whether or not any protein mediates the effect of copper is currently unknown).

the third gene of the light-inducible carQRS operon. Protein CarS does not bind DNA, but physically interacts with CarA to dismantle the CarA-DNA complex (RuizVázquez et al., 1993; Whitworth \& Hodgson, 2001; Cervantes \& Murillo, 2002; López-Rubio et al., 2002; Pérez-Marín et al., 2004; Navarro-Avilés et al., 2007).

The third protein required early for the activation of both crtI and the $c a r B$ operon is the product of the unlinked $c a r F$ gene. Strong genetic evidence indicates that CarF is the earliest acting of all known Car elements of the light-driven regulatory cascade, being absolutely necessary for the lightdependent inactivation of CarR (Fontes et al., 2003). Contrary to all other known Car regulatory proteins, CarF does not take part in the carotenogenic response to copper (Moraleda-Muñoz et al., 2005). CarF shows sequence similarity to members of a family of proteins named Kua. When first described, this family only included eukaryotic proteins (Fontes et al., 2003), but some prokaryotic members of the Kua family have now been identified (see below). At present, none of the Kua proteins has a known function.

In the present work, we have investigated the mode of action of protein CarF, with a focus on the subcellular localization of CarF and on whether the functional interaction with CarR uncovered by genetic data is mediated by a physical association between the two proteins. The results reported here strongly argue for CarF acting as a membrane-bound anti-antisigma factor and serve to determine a series of protein domains and histidine residues that are essential for normal CarF activity.

\section{METHODS}

Bacterial strains, yeast strains and growth conditions. The following Escherichia coli strains were used: DH5 $\alpha$ (Hanahan, 1983) for cloning procedures, BL21 ( $2 \mathrm{DE} 3$ ) for protein overexpression, and JM109 (Yanisch-Perron et al., 1985) and BTH101 (adenylate-cyclase deficient) for a bacterial two-hybrid system (BACTH; Karimova et al., 1998, 2000). The host Saccharomyces cerevisiae strains for the yeast two-hybrid systems were EGY48 (for the LexA-based system; Golemis \& Serebriiskii, 2001) and cdc25H (for the Cytotrap system; Stratagene). Yeast growth media and conditions were as described elsewhere (Ausubel et al., 1988). M. xanthus strain DK1050 (RuizVázquez \& Murillo, 1984) was the wild-type strain used in this study. The M. xanthus carF2 deletion mutant MR992 (Fontes et al., 2003) was also used. The rich casitone-tris (CTT) medium and the exact culture conditions for growth of $M$. xanthus in the dark and in the light have been described previously (Bretscher \& Kaiser, 1978; Fontes et al., 1993).

Nucleic acid manipulations. Standard protocols were followed for DNA manipulation (Sambrook \& Russell, 2001). For DNA amplification (PCR) three different systems were used, depending on the aim of the experiment. For amplification of short DNA fragments, $P f u$ high-fidelity DNA polymerase (Promega) was employed. For diagnostic PCR (i.e. in the analysis of strains carrying deleted versions of $(a r F)$, the Amersham Ready To Go PCR kit was used. For inverse PCR (i.e. in the generation of the Ser86-Ser139 internal deletion of CarF), the Roche Long Template High-Fidelity PCR system was used. Reaction mixtures were supplemented with $5 \%(\mathrm{v} / \mathrm{v})$ DMSO to overcome PCR difficulties due to the high $\mathrm{G}+\mathrm{C}$ content of the template DNA. Each PCR-derived clone was verified by sequencing.

Site-directed mutagenesis. The technique of PCR overlap extension (Ho et al., 1989) was used for the site-directed substitution of CarF His164 and His195 residues by alanine (for details see Fontes et al., 2003). The mutagenic primers used were His164Fw (5'-GCGACCAACCAGTTCGCCAAGTGGTCGCACATG-3') and His164Rev (5'-CATGTGCGACCACTTGGCGAACTGGTTGGTCGC-3') for the H164A substitution; and His $\overline{195} \mathrm{Fw}$ (5'-CACCACCGCATTCACGCCACCAAGCCGTACAAC-3') and His195Rev (5'-GTTGTACGGCTTGGTGGCGTGAATGCGGTGGTG-3') for the H195A substitution (nucleotides that differ from the wild-type sequence are underlined). The products from the overlap extension, done with primers that amplify the complete $c a r F$ ORF, were cloned separately into the EcoRI site of plasmid pMAR979. This plasmid contains the carF genomic region but lacks the carF ORF, which has been substituted by an EcoRI site (Fontes et al., 2003). In independent experiments, each plasmid with the mutant carF allele was electroporated into the carF2 deletion mutant strain MR992 (Fontes et al., 2003). As the plasmid cannot replicate in $M$. xanthus, $\mathrm{Km}^{\mathrm{R}}$ colonies arise from an 
integrative, homologous recombination event between the M. xanthus DNA in the pMAR979-derived plasmid and the homologous chromosomal region in the recipient strain. The correct integration of every construction in the genome was tested by PCR. To obtain carF alleles that lacked the $\mathrm{N}$ - or the C-terminal region, the corresponding PCR fragments were inserted into the EcoRI site of pMAR979. To obtain a CarF version lacking the region between amino acids 86 and 139, we used inverse PCR to amplify all the region flanking the fragment to be deleted, using a plasmid with $7.7 \mathrm{~kb}$ of the wild-type $c a r F$ region as template. The plasmids were introduced into the MR992 carF2 deletion mutant or into the wild-type strain as described above. Every plasmid was verified by sequencing.

CarF overexpression and purification. The carF ORF was amplified by PCR from $M$. xanthus genomic DNA and cloned into the NdeI and BamHI sites of pET11b to obtain plasmid pET11b-carF (Studier et al., 1990). Cells transformed with pET11b-carF were grown in $50 \mathrm{ml} \mathrm{LB} /$ ampicillin/glucose at $37{ }^{\circ} \mathrm{C}$ to an $\mathrm{OD}_{600}$ of $0.6-$ 1.0 , harvested by centrifugation, washed and inoculated into 11 of the same medium without glucose. After growing to an $\mathrm{OD}_{600}$ of $0.6-0.8$, protein expression was induced with $0.4 \mathrm{mM}$ IPTG overnight at $25{ }^{\circ} \mathrm{C}$. SDS-PAGE analysis of cell extracts showed that CarF was expressed mainly as an insoluble protein. Cells from 11 induced culture were pelleted, lysed by mechanical treatment with alumina at $4{ }^{\circ} \mathrm{C}$ and resuspended in $30 \mathrm{ml}$ buffer A $(50 \mathrm{mM}$ Tris, $5 \%$, v/v, glycerol, $5 \mathrm{mM} \beta$-mercaptoethanol, pH 7.5) containing $300 \mathrm{mM}$ $\mathrm{NaCl}, 1 \mathrm{mM}$ PMSF and $1 \mathrm{mM}$ benzamidine. Resuspended cells were subjected to sonication under ice-cold conditions and the alumina was eliminated by low-speed centrifugation $\left(500 \mathrm{~g}, 5 \mathrm{~min}, 4^{\circ} \mathrm{C}\right)$. The supernatant was then centrifuged $\left(12000 \mathrm{~g}, 15 \mathrm{~min}, 4^{\circ} \mathrm{C}\right)$ to isolate inclusion bodies and the pellet was solubilized in denaturing buffer (8 $\mathrm{M}$ urea, $300 \mathrm{mM} \mathrm{NaCl}, 77 \mathrm{mM} \mathrm{Na} \mathrm{HPO}_{4}, 22.6 \mathrm{mM} \mathrm{NaH}_{2} \mathrm{PO}_{4}$, $\mathrm{pH}$ 7.6) and insoluble material was eliminated by centrifugation $\left(12000 \mathrm{~g}, 15 \mathrm{~min}, 4{ }^{\circ} \mathrm{C}\right)$. The supernatant was used for CarF purification by TALON (Clontech) metal affinity resin under denaturing conditions, followed by imidazole elution $(150 \mathrm{mM})$. Plasmid pET11b is designed for the overexpression of native proteins, but the abundance of histidine residues in CarF enables CarF purification by using a nickel resin.

Obtention of anti-CarF antibodies. The denatured CarF protein was gel-purified and employed to raise a rabbit polyclonal antiserum. Western blotting confirmed that the unpurified antiserum recognized the pure CarF protein and the protein expressed in E. coli cells carrying pET11b-carF. No signal was detected when a preimmune antiserum was used against the same samples (data not shown).

Isolation and solubilization of membranes from $E$. coli. The pellet from a $250 \mathrm{ml}$ culture of E. coli transformed with pET11b-carF was treated as described above for CarF purification. The resulting supernatant was then centrifuged at $90000 \mathrm{~g}$ for $45 \mathrm{~min}$ at $4{ }^{\circ} \mathrm{C}$. The supernatant was retained, as this contained the cytoplasmic and periplasmic proteins (soluble fraction). The pellet (containing membranes) was washed with cold buffer $\mathrm{A}$ and resuspended in $4 \mathrm{ml}$ of the same buffer with $1 \%$ Triton X-100, $1 \mathrm{mM}$ benzamidine and $1 \%$ protease inhibitor mixture (Sigma). The mixture was incubated for $1 \mathrm{~h}$ at $4{ }^{\circ} \mathrm{C}$ with shaking and centrifuged at $90000 \mathrm{~g}$, as before, to separate the solubilized membrane (supernatant) from the non-solubilized membrane (pellet). The protein concentration of each fraction was determined using the Bio-Rad protein assay kit, as specified by the manufacturer. To detect CarF, $6 \mu \mathrm{g}$ protein from each sample was subjected to Western analysis with anti-CarF antibodies (Cayuela et al., 2003).

Sequence analysis and topological prediction. For comparison of CarF with databases, the WU-BLASTP + BEAUTY (http://searchlauncher. bcm.tmc.edu/seq-search/protein-search.html) and BLAST2-wU (www.
ebi.ac.uk/Tools/similarity.html) programs, provided by the BCM search launcher and EBI, respectively, were used. For the alignment of different peptides or proteins, the program CLUSTALW 1.8 (http:// searchlauncher.bcm.tmc.edu/multi-align/multi-align.html) from BCM was employed. For the prediction of transmembrane regions and CarF topology, the programs TMPRED and SOSUI from the BCM search launcher (http://searchlauncher.bcm.tmc.edu/seq-search/struc-predict. html) and the programs TMHMMFIX (www.sbc.su.se/ melen/ TMHMMfix/) and DAS (www.sbc.su.se/ miklos/DAS/maindas.html) from the SBC server were used.

Analysis of $\boldsymbol{\beta}$-galactosidase. Qualitative determination of $\beta$ galactosidase was performed on LB plates containing $40 \mu \mathrm{g}$ X-Gal $\mathrm{ml}^{-1}$. Quantitative analysis on liquid cultures was performed as described previously (Balsalobre et al., 1987). Enzyme-specific activities are given in $\mathrm{nmol} o$-nitrophenol hydrolysed $\min ^{-1}(\mathrm{mg}$ protein $)^{-1}$. For analysis of protein interactions with the BACTH system, overnight cultures, grown at $30{ }^{\circ} \mathrm{C}$ in $\mathrm{LB}$ medium with the appropriate antibiotics, were diluted and incubated up to $\mathrm{OD}_{550}$ $\sim 0.5$. At that point, IPTG $(0.5 \mathrm{mM})$ was added and the cultures were incubated for $8 \mathrm{~h}$ at $30^{\circ} \mathrm{C}$.

\section{RESULTS}

\section{CarF is located at the membrane in $E$. coli}

Lack of the carF gene product blocks the M. xanthus carotenogenic response to blue light ( $\mathrm{Car}^{-}$phenotype), whereas lack of the carR gene product causes constitutive, light-independent carotenoid synthesis ( $\mathrm{Car}^{\mathrm{C}}$ phenotype). The fact that carF carR double mutants show the same $\mathrm{Car}^{\mathrm{C}}$ phenotype as the carR single mutant strongly suggests that CarF participates in the light-mediated inactivation of CarR (Fontes et al., 2003; Fig. 1). CarR has been shown to be an integral membrane protein (Gorham et al., 1996; Browning et al., 2003), as may also be the case with CarF, since its amino acid sequence predicts at least three transmembrane domains (Fontes et al., 2003). To test this, we purified CarF from E. coli cells carrying plasmid pET11b-carF, and polyclonal anti-CarF antibodies were raised (see Methods). CarF was easily detected by Western analysis of the producing $E$. coli strain, but could not be detected by the same analysis of the $M$. xanthus wild-type strain DK1050, or the protease-deficient strain DK5077 (Petit \& Guespin-Michel, 1992). To determine the subcellular localization of CarF in E. coli, cell lysates were prepared from mid-exponential-phase cultures overexpressing $\operatorname{carF}$ (see Methods). High-speed centrifugation was used to separate a soluble fraction (containing proteins from the cytoplasm and the periplasm) from an insoluble particulate fraction (containing cell membranes, wall components and ribosomes). The insoluble fraction was washed and incubated with $1 \%$ Triton X-100. The solubilized membrane components were separated from the non-solubilized membrane fraction by high-speed centrifugation. As a control, the same procedure was performed with cultures of $E$. coli carrying vector pET11b. Aliquots of each fraction (containing the same amount of total protein) were subjected to Western blotting and probed with polyclonal anti-CarF antibodies (Fig. 2). A band 

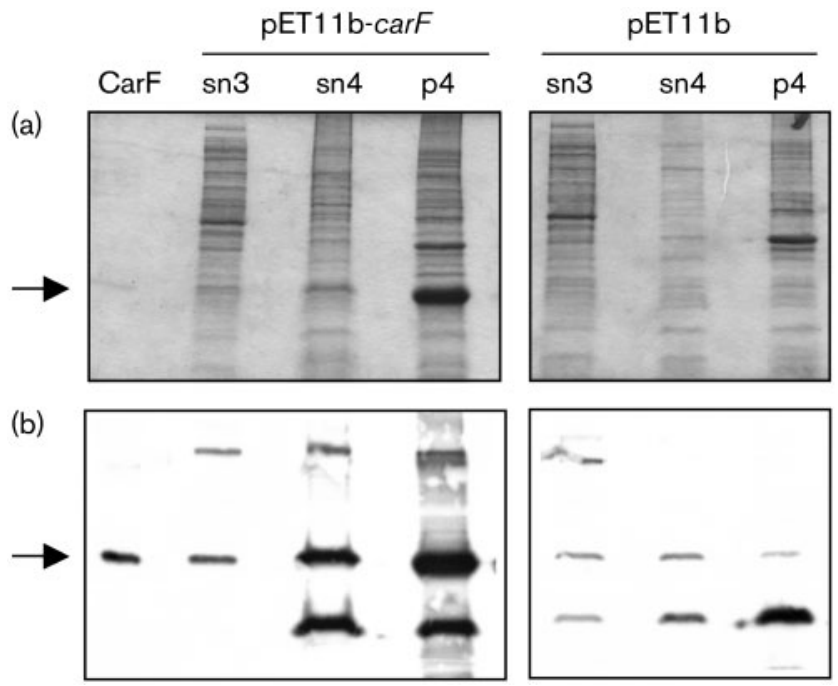

Fig. 2. Subcellular localization of the CarF protein in E. coli BL21 cell extracts carrying plasmids $\mathrm{pET} 11 \mathrm{~b}$-carF (left side) or $\mathrm{pET} 11 \mathrm{~b}$ (right side). (a) Coomassie staining. (b) Western blot using antiCarF antiserum. CarF, purified CarF protein; sn3, soluble cytoplasm and periplasm proteins; sn4, $1 \%$ Triton X-100 soluble proteins from plasma membrane; $\mathrm{p} 4,1 \%$ Triton $\mathrm{X}-100$ insoluble proteins. The arrows indicate the position of the CarF protein (approx. $30 \mathrm{kDa}$ ).

of approximately $30 \mathrm{kDa}$ (the expected size for CarF) was observed in the solubilized and non-solubilized membrane fractions. This indicates that CarF accumulates in the membrane and that it can be partially solubilized with $1 \%$ Triton X-100. The anti-CarF serum recognizes other E. coli proteins, probably due to impurities in the preparation of the CarF protein used to raise the antibodies (Fig. 2).

\section{Membrane topology of CarF}

Amino acid sequence analysis of CarF with different programs to predict transmembrane regions (TMPRED, TMHMM, SOSUI and DAS) suggests the presence of either three or four transmembrane domains in CarF. To assess the topology of CarF and the actual number of transmembrane domains, we used the pKY481 vector, which allows in-frame cloning of DNA fragments immediately upstream of the lac $Z$ gene to make translational fusions (Cho \& Zusman, 1999). Five different M. xanthus DNA fragments, each encoding a CarF protein containing one, two, three or four putative transmembrane domains, or the whole protein, were cloned into pKY481 (Fig. 3). $\beta$ Galactosidase is active only in the cytoplasm, as it is inhibited by the high $\mathrm{pH}$ of the periplasm (Gött \& Boos, 1988). Thus, a positive result in the assay for $\beta$ galactosidase activity would indicate that the $\mathrm{C}$ terminus of a particular protein fusion faces the cytoplasm.

Previous experiments had revealed that the $\operatorname{car} F$ promoter is not active in E. coli. Thus, to express the translational
CarF-LacZ fusions, we employed the promoter of the $M$. xanthus gene phoR1 that yields measurable activity in E. coli (Carrero-Lérida et al., 2005). A 781 bp fragment containing the phoR1 promoter was amplified by PCR from genomic $M$. xanthus DNA and cloned upstream of every one of the carF-lacZ fusions. The five constructions, and the vector pKY481 alone, were separately transferred into E. coli. The results of the $\beta$-galactosidase assays (Fig. 3) indicate that CarF might indeed contain four transmembrane domains. The first and third transmembrane domains of CarF should be oriented towards the periplasmic space, as LacZ fusions at amino acids 56 and 142 yielded only basal $\beta$-galactosidase activity. On the contrary, the second and fourth transmembrane motifs should be oriented to the cytoplasm, as fusions to amino acids 83 and 170 produced significantly higher $\beta$ galactosidase activities. It should be noticed that the $\beta$ galactosidase activity found when the entire CarF protein was fused to LacZ is similar to the activity obtained by the fusion to the fourth transmembrane motif.

\section{Analysis of CarF-CarR interaction using yeast- based two-hybrid systems}

As previously mentioned, genetic data points to CarF being involved in the light-driven inactivation of CarR (Fontes et al., 2003). The functional interaction between CarF and CarR might be mediated by a physical association between the two proteins. So, the yeast two-hybrid system described by Golemis \& Serebriiskii (2001) was tried to assay a possible CarF-CarR physical interaction. Both CarF and CarR were used either as a 'bait' (fused to the DNAbinding domain of LexA) or as a 'target' (fused to the yeast activation domain B42) in independent experiments. In neither case did the bait constructions enter the nucleus of the yeast cell, probably due to the hydrophobic nature of both CarF and CarR. We then tested different, nonhydrophobic segments of CarF and CarR, but none of the bait fusions entered the yeast nucleus, maybe due to the instability of the peptides and/or their degradation by yeast proteases.

We also tested the putative interaction between CarF and CarR by employing the Cytotrap yeast two-hybrid system (Stratagene). In this system, one protein fusion serves to anchor a 'target' protein to the membrane. Upon interaction, the second protein fusion ('bait') is brought to the membrane to activate the Ras signalling pathway. In our case, both CarF and CarR, when used as 'baits', activated Ras signalling, even in the absence of the 'target' protein fusion.

\section{Analysis of protein interactions using a BACTH system}

Due to the restrictions of yeast two-hybrid systems to detect interaction between hydrophobic proteins, we employed a BACTH system (Karimova et al., 1998). This 

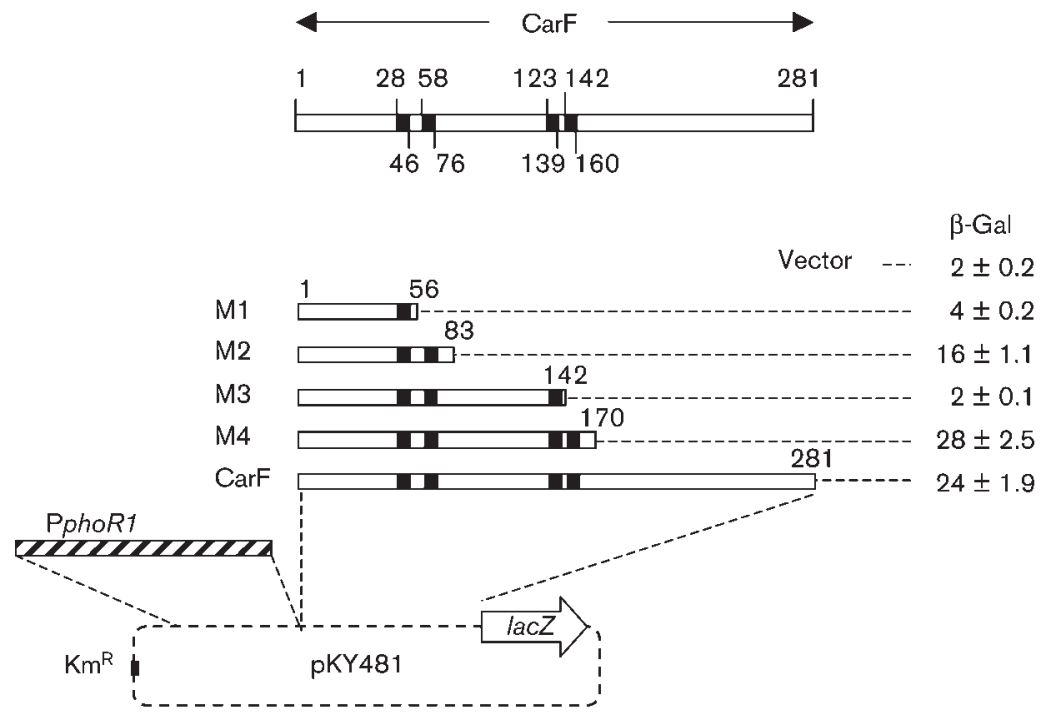

Fig. 3. Topological in vivo analysis of the CarF protein in the E. coli membrane. Different carF fragments were cloned downstream of the phoR1 promoter and upstream of the lac $Z$ gene of vector pKY481 to make translational fusions. Predicted transmembrane domains are indicated in black. The position of the amino acid located at the end of each CarF fragment is indicated. $\beta$-Galactosidase activity is given in $\mathrm{nmol} \mathrm{o}$-nitrophenol hydrolysed $\min ^{-1}$ (mg protein) ${ }^{-1}$. Data are means $\pm S D$ of at least three independent experiments.

system is based on the reconstitution of the cAMP signal transduction pathway in an E. coli adenylate-cyclasedeficient $\left(\mathrm{cya}^{-}\right)$strain. It takes advantage of the modular structure of the catalytic domain of the Bordetella pertussis adenylate cyclase, which consists of two complementary fragments (T25 and T18). When separately expressed in E. coli, they do not form an active enzyme, unless interacting polypeptides are genetically fused to both fragments. Restoration of enzymic activity results in cAMP synthesis and activation of the expression of several resident E. coli genes (e.g. lac, mal). The carF and carR genes were cloned into the vectors pKT25, pUT18 and pUT18C. In pUT18, the protein under study is cloned at the $\mathrm{N}$-terminal end of the T18 polypeptide, whereas in pUT18C it is fused to its C-terminal end. The resulting plasmids (pUT18carF, pUT18CcarF, pKT25carF, pUT18carR, pUT18CcarR and pKT25carR) were used in pairs to transform the E. coli cya $^{-}$strain BTH101 and the transformants were plated onto LB-X-Gal and MacConkey-maltose media. Those transformants carrying simultaneously a carR and a carF fusion, either to the T18 or the T25 complementing fragments, showed a LacZ ${ }^{+} \mathrm{Mal}^{+}$phenotype, in contrast to their respective self-activation controls. The results of quantitative $\beta$-galactosidase assays of the different transformants, shown in Fig. 4(a), confirmed that CarF and CarR physically interact with each other. Stronger interactions were consistently observed when both CarF and CarR were fused to the N-terminal end of the T18 fragment, leaving their own N-terminal ends free. The CarF-CarR interaction in $E$. coli is light-independent, as the $\beta$ galactosidase activities of the cultures grown in the dark were similar to those grown in the light (data not shown).

Browning et al. (2003) showed that CarR is capable of forming homomultimers, as other antisigma factors do (Campbell \& Darst, 2000). The same could happen to CarF. Thus, we tested for CarF-CarF interaction by employing the BACTH system, and also used this system to corroborate the CarR-CarR interaction. The $\mathrm{LacZ}^{+}$ $\mathrm{Mal}^{+}$phenotype and the $\beta$-galactosidase specific activities generated by the appropriate constructions revealed that, as for CarR, different molecules of CarF can interact with each other (Fig. $4 \mathrm{~b}$ and c).

The stoichiometry of CarQ and CarR is critical for the regulation of carQRS expression. This and other data suggest that, in the dark, CarQ and CarR might form a complex in the membrane (Martínez-Laborda \& Murillo, 1989; Gorham et al., 1996; Browning et al., 2003). To test for CarQ-CarR interaction, Browning et al. (2003) used a yeast two-hybrid system. They could detect interaction only between some fragments of CarQ and CarR, but not between intact CarQ and CarR proteins. This could be due to the limitations of the yeast two-hybrid system for testing hydrophobic proteins. We have now used the BACTH system to test for interaction between intact CarQ and CarR. The carQ ORF was amplified by PCR and separately cloned into vectors pUT18C and pKT25. The resulting plasmids (pUT18CcarQ and pKT25carQ) were separately introduced into E. coli, in combination with pUT18carR, pUT18CcarR or pKT25carR. The LacZ ${ }^{+} \mathrm{Mal}^{+}$phenotypes and the $\beta$-galactosidase specific activities of the resulting transformants revealed that intact CarQ and CarR can interact with each other. The interaction was particularly strong when the CarQ C-terminal end remained free (Fig. 4d). The CarQ-CarR interaction in E. coli was also light-independent, as similar $\beta$-galactosidase specific activities were observed in cultures grown in the dark or in the light (data not shown).

Finally, the BACTH system was also used to test for a possible direct interaction between CarF and CarQ. No sign of interaction was detected in transformants carrying the pairs of plasmids pUT18CcarQ+pKT25carF, pUT18carF + pKT25carQ or pUT18CcarF + pKT25carQ (data not shown). 
(a)

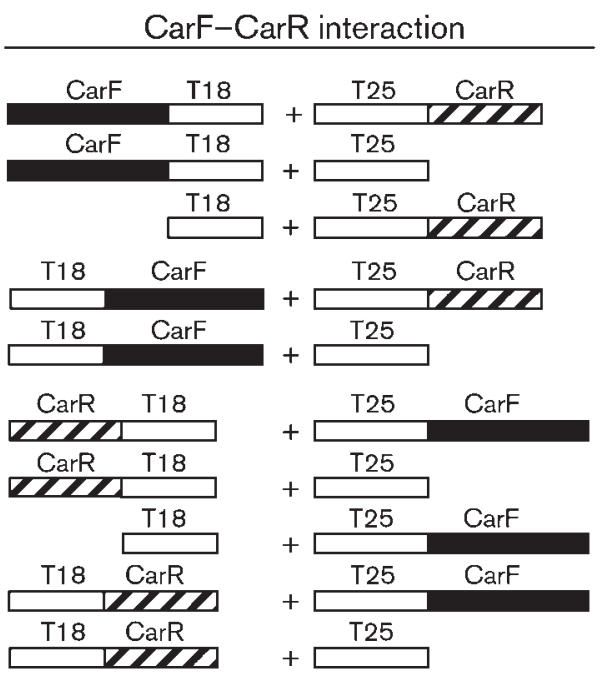

(b)

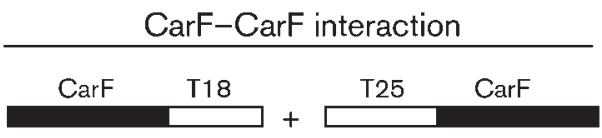

(c)

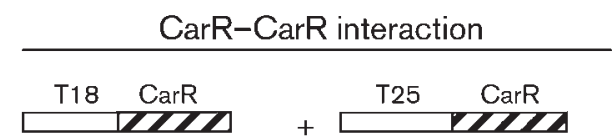

(d)

\begin{tabular}{|c|c|c|c|}
\hline \multicolumn{4}{|c|}{ CarQ-CarR interaction } \\
\hline $\mathrm{T} 18$ & 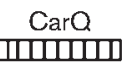 & $\mathrm{T} 25$ & CarR \\
\hline CarR & T18 & $\begin{array}{r}\text { T25 } \\
+\square\end{array}$ & سمبس \\
\hline $\begin{array}{r}\mathrm{T} 18 \\
\end{array}$ & CarR & $\mathrm{T} 25$ & $\begin{array}{c}\text { CarO } \\
\text { IIIIIII }\end{array}$ \\
\hline
\end{tabular}

$\beta$-Galactosidase

$2853 \pm 207$

$78 \pm 5$

$83 \pm 6$

$528 \pm 34$

$91 \pm 2$

$962 \pm 73$

$76 \pm 4$

$96 \pm 7$

$299 \pm 19$

$100 \pm 6$

$650 \pm 27$

$3500 \pm 275$

Fig. 4. Analysis of protein interactions using the BACTH system. The bait and target constructions employed for the interaction assays and their $\beta$-galactosidase activities [in nmol o-nitrophenol hydrolysed $\mathrm{min}^{-1}$ (mg protein $)^{-1}$ ] are shown. The values of the selfactivation controls are also shown. Data are means $\pm S D$ of at least three independent experiments.

\section{CarF truncated versions}

Comparative analysis of the predicted CarF protein sequence $(281 \mathrm{aa})$ with other proteins in the databases shows that CarF shares strong similarity to members of a family of proteins, known as Kua, whose function is unknown (Fontes et al., 2003). These proteins were originally found only in eukaryotic organisms, but some prokaryotic members have been described recently (see supplementary Fig. S1 available with the online version of this paper). Most Kua proteins share several conserved domains, located between hydrophobic regions, whose importance was studied by generating truncated versions of carF and analysing their ability to phenotypically complement the deletion of $\operatorname{carF}$ (Fig. 5).

Three fragments of the $c a r F$ gene were amplified by PCR and cloned into the EcoRI site of vector pMAR979, which carries a deletion of the entire carF ORF (Fontes et al., 2003; see Methods). The resulting constructions contained one N-terminal deletion (Met1-Ala25) and two C-terminal deletions (Val160-Pro281 and Gly241-Pro281). In addition, an internal deletion (from Ser86 to Ser139) was constructed by inverse PCR (Fig. 5; see Methods for details). The small C-terminal region deleted in pMAR2742 is not conserved in other Kua proteins, neither does it show similarity to other proteins or protein domains in the databases. The N-terminal region deleted in plasmid pMAR2728 shows a moderate conservation among Kua proteins. The large C-terminal region deleted in pMAR2731 and the internal region deleted in pMAR2727 contain sequences highly conserved in Kua proteins.

All indicated carF deletions were separately introduced into the M. xanthus carF mutant strain MR992, and more than 100 independent colonies from each transformation were tested for their Car phenotype. All colonies showed a $\mathrm{Car}^{-}$ phenotype, except for those that had integrated the CarF version lacking the $\mathrm{C}$-terminal 41 amino acids (Fig. 5). The $\mathrm{Car}^{+}$phenotype of these latter colonies was indistinguishable from that of the M. xanthus wild-type strain DK1050. 

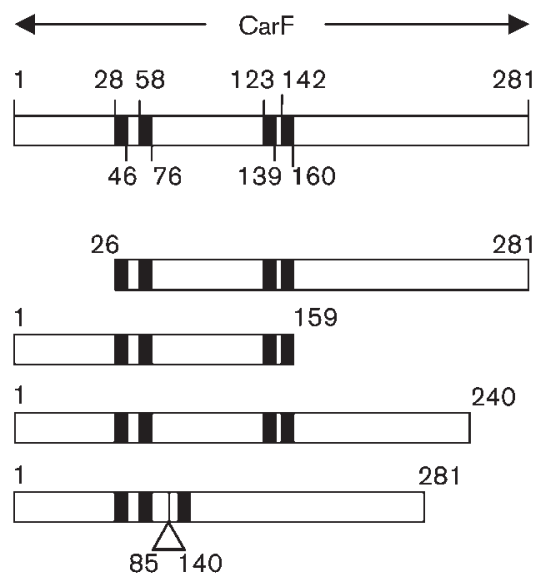

\begin{tabular}{llll} 
& MR992 & & DK1050 \\
\cline { 2 - 2 } pMAR2728 & Car & & Car $^{+}$ \\
pMAR2731 & Car & & Car $^{+}$ \\
pMAR2742 & Car $^{+}$ & & Car $^{+}$ \\
pMAR2727 & Car & Car $^{+}$
\end{tabular}

Fig. 5. Car phenotype of truncated versions of CarF in the MR992 ( $\Delta$ carF) and DK1050 (wt) backgrounds. The position of the first and the last amino acid of each polypeptide (and of the internal deletion) are indicated. Dark bars symbolize the transmembrane domains.

Therefore, truncation of the fragments Met1-Ala25, Val160-Pro281 and Ser86-Ser139 affects CarF activity, conformation or stability, whereas the last 41 amino acids of CarF are not essential for the correct function of the protein. When the truncated versions of CarF were introduced into the wild-type strain DK1050, all the tested transformants accumulated carotenoids in the light like the parental strain. The dominance of the wild-type carF allele over the mutated ones indicates that none of the truncated CarF proteins can interfere with the normal action of wildtype CarF.

\section{Substitution of conserved His to Ala residues}

Comparison of the amino acid sequence of CarF with other Kua proteins shows a remarkable conservation of several His residues (supplementary Fig. S1). Eight of the 12 His residues of CarF are present, at the same relative position, in all Kua proteins. Three of the other His residues are present only in the animal Kua proteins, some of the prokaryotic ones, and that from Leishmania major. The remaining His residue is found exclusively in CarF. Previous experiments had shown that three of those conserved residues (His78, His103 and His113) are essential for the normal activity of CarF, while the nonconserved His218 is not (Fontes et al., 2003).

We have extended the analysis of the functional relevance of CarF His residues by making His to Ala substitutions at positions 164 and 195, which are conserved in all Kua proteins (supplementary Fig. S1). Site-directed mutagenesis was performed by PCR overlap extension (see Methods) and the plasmids carrying the carF gene with the desired substitutions were transformed separately into the M. xanthus carF deletion mutant MR992. In each case, more than 100 transformants were tested for the colour phenotype. All the colonies carrying the H164A or H195A substitutions showed the same $\mathrm{Car}^{-}$phenotype as the parental strain MR992. Thus, the conserved His164 and His195 residues are essential for the normal activity of CarF, as shown with the previously tested conserved residues His78, His103 and His113. Substitutions H164A and H195A were also introduced separately into the $M$. xanthus wild-type strain. In both cases, the transformants were $\mathrm{Car}^{+}$, except for a few colonies $(1 \%)$ that showed a $\mathrm{Car}^{-}$phenotype, probably the consequence of gene conversion (Stephens \& Kaiser, 1987; Martínez-Laborda \& Murillo, 1989).

\section{DISCUSSION}

Cells of the bacterium M. xanthus are known to accumulate carotenoid pigments only when stimulated by exposure to blue light or copper. Both responses depend on the activation at the transcriptional level of the structural genes for carotenoid synthesis. Our present model on how this is accomplished, based on many genetic and biochemical studies (see references in the Introduction), is summarized in Fig. 1. Both light- and copper-induced activation of the carotenogenic genes depend crucially on the inactivation of CarR, an antisigma factor that in the dark sequesters the ECF-sigma factor CarQ to the membrane. At present, nothing is known on how exposure to copper leads to inactivation of CarR. As for the light response, we have previously reported strong genetic evidence for CarR inactivation requiring the product of the $M$. xanthus gene carF, the only known element of the carotenogenic regulatory cascade that does not participate in the response to copper (Fontes et al., 2003; Moraleda-Muñoz et al., 2005).

To detect CarF, we raised anti-CarF antibodies. Unfortunately, we could not detect CarF by Western analysis of $M$. xanthus cell extracts. The low activity of the carF promoter may have contributed to our failure to detect CarF in M. xanthus, as we could easily detect the protein in the overproducing E. coli strain. A similar situation has been reported precisely for the $M$. xanthus antisigma factor CarR, which could not be detected by Western analysis neither in the wild-type nor in a strain in which expression of CarR was sixfold greater than in the wild-type (Browning et al., 2003). 
In silico sequence analysis predicts the presence in CarF of several transmembrane domains (Fontes et al., 2003; see also supplementary Fig. S1). Two lines of evidence presented in this paper confirm that CarF is a membrane protein. On the one hand, when expressed in E. coli, CarF accumulates almost exclusively in the membrane, from where it can be only partially solubilized with the detergent Triton X-100 (Fig. 2). On the other hand, a lacZ-based topological test for potential transmembrane domains detects four transmembrane domains in CarF, the first and third being oriented towards the periplasm, and the second and fourth towards the cytoplasm (Fig. 3). The fact that CarF alone, like CarR, led to Ras signalling when used as bait in the Cytotrap two-hybrid system (Stratagene) further confirms its membrane localization.

As mentioned before, CarR has been shown to be a membrane protein that sequesters the ECF-sigma factor CarQ in the dark. A previous study of CarR-CarQ interaction, using a yeast two-hybrid system that requires the fusion proteins to enter the nucleus, gave positive results only with some fragments of the proteins involved (Gorham et al., 1996; Browning et al., 2003). Our own results with the Cytotrap system mentioned above corroborate that CarR goes to the membrane when expressed in yeast cells. To test for protein interactions, we have used a BACTH system (Karimova et al., 1998, 2000) that, in principle, should work well with membrane proteins. As shown in Fig. 4(a, d), the bacterial test produced unequivocal evidence that CarF interacts physically with CarR, and corroborated that complete CarR and CarQ also interact physically with each other. Other results of the bacterial test indicate that CarF can form homomultimers (Fig. 4b), as does CarR (Fig. 4c). It should be noted that no interaction between CarF and CarQ was observed in the bacterial test.

Considering previous genetic data on the role of CarF in the light-activated regulatory cascade of $M$. xanthus (Fontes et al., 2003) and its physical interaction with CarR uncovered in this work, we propose that CarF is in fact an anti-antisigma factor for CarR. The interplay of the M. xanthus proteins CarF, CarR and CarQ reminds one of the Bacillus subtilis SpoIIAA, SpoIIAB and $\sigma^{\mathrm{F}}$ proteins, which have been subjected to extensive genetic and molecular analysis (for a review see Yudkin \& Clarkson, 2005). In this case, a dimer of SpoIIAB binds a single molecule of $\sigma^{\mathrm{F}}$ and prevents it from acting as a transcription factor. During the process of sporulation, SpoIIAA binds the $\sigma^{\mathrm{F}}$-SpoIIAB ${ }_{2}$ complex and causes the release (activation) of $\sigma^{\mathrm{F}}$. As appears to happen with CarF, SpoIIAA does not interact physically with its cognate sigma factor, but docks on the $\sigma^{\mathrm{F}}$-SpoIIAB ${ }_{2}$ complex by binding a subunit of SpoIIAB that is not in contact with $\sigma^{\mathrm{F}}$ (Ho et al., 2003).

Sequence comparison analysis gave no hint of what the molecular mechanism for CarF might be, and how it could be modulated by the light signal. When first described,
CarF was reported to be very similar to a family of proteins of unknown function, named Kua, found only in eukaryotes (Thomson et al., 2000; Fontes et al., 2003). In a recent search, we have identified Kua-like proteins, also of unknown function, in prokaryotes and even in a virus (see supplementary Fig. S1). Transmembrane domains similar to those found in CarF are predicted for most Kua proteins. As seen in Fig. 6 and supplementary Fig. S1, the most conserved amino acid residues are grouped in two regions, one between the second and third transmembrane domains and another at the $\mathrm{C}$ terminus, following the fourth transmembrane domain. The importance of these conserved regions for the action, or stability, of CarF has been corroborated here by the inability of various truncated versions of CarF to complement a carF deletion. Singular to CarF among the Kua proteins is a C-terminal extension of about 40 aa. These appear to be dispensable, as deleting them does not affect CarF function in a significant way (Fig. 5).

Previous theoretical analysis revealed some similarities, but no overall sequence similarity, between Kua proteins and fatty acid desaturases and hydroxylases (Los \& Murata, 1998; Thomson et al., 2000; Fontes et al., 2003). These enzymes contain two transmembrane domains that make the protein cross the membrane twice, and eight His residues, located at cytoplasmic domains, all of which play a critical role in the reaction mechanism (Mitchell \& Martin, 1997; Shanklin et al., 1994). The topological assays carried out in this work show that CarF contains four transmembrane domains instead of two. The four transmembrane domains could be grouped in two pairs. In each one of them, one helix would face the periplasm and the other, only a few residues apart, would face the cytoplasm. Prominent among the conserved Kua residues are eight His residues that are found in all members of the family

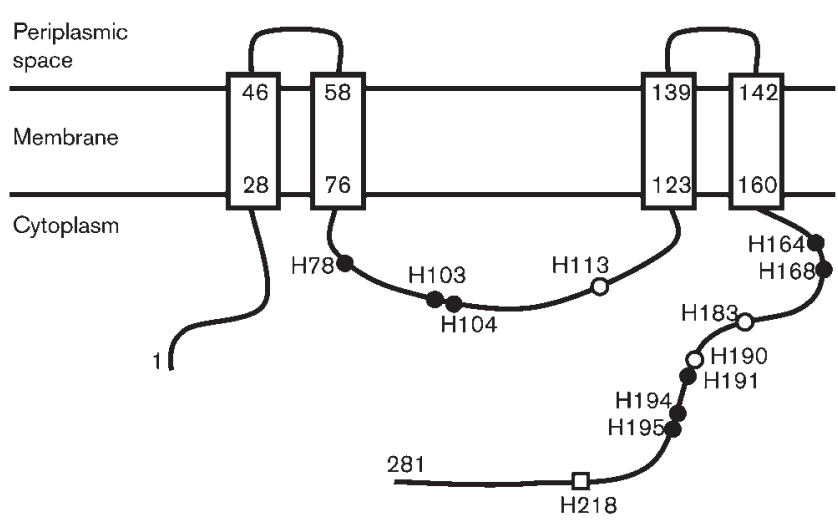

Fig. 6. Membrane topology model of CarF. Five non-hydrophobic domains are interrupted by four transmembrane motifs (drawn as rectangles). The histidine residues found in all Kua proteins are shown as black circles; the histidine residues that are conserved in at least $50 \%$ of the Kua proteins are marked with white circles; His218 (only found in CarF) appears as a white square. 
(supplementary Fig. S1), and they, as for the enzymes mentioned above, would be located at the cytoplasm (Fig. 6). All single His to Ala substitutions at five of the eight conserved His residues (His164 and His195, reported here, and His78, His103 and His113, reported by Fontes et al., 2003) result in the inactivation of CarF. For one of the hydroxylases mentioned above, it has been shown that the multiple His residues serve to form a catalytically essential di-iron cluster (Shanklin et al., 1997). The ability of CarF to bind transition metals is revealed by its ability to adsorb, in untagged form, $\mathrm{Co}^{2+}$ resins. However, as shown with other membrane proteins, purification of CarF in its native form has proven problematic. Certainly, more work will be needed to search for a possible prosthetic group in CarF and to unravel its possible role in triggering its antiantisigma activity under blue light.

\section{ACKNOWLEDGEMENTS}

We thank J. A. Madrid for technical assistance. This work was supported by grants BCM2003-00658 and BFU2006-14524 from the Ministerio de Educación y Ciencia (Spain), and a fellowship to L. G.-M. from Fundación Séneca-Murcia (Spain).

\section{REFERENCES}

Ausubel, F. M., Brent, R., Kingston, R., Seidman, J. G., Smith, J. A. \& Struhl, K. (1988). Current Protocols in Molecular Biology. New York: John Wiley \& Sons.

Balsalobre, J. M., Ruiz-Vázquez, R. M. \& Murillo, F. J. (1987). Light induction of gene expression in Myxococcus xanthus. Proc Natl Acad Sci U S A 84, 2359-2362.

Botella, J. A., Murillo, F. J. \& Ruiz-Vázquez, R. (1995). A cluster of structural and regulatory genes for light-induced carotenogenesis in Myxococcus xanthus. Eur J Biochem 233, 238-248.

Bretscher, A. P. \& Kaiser, D. (1978). Nutrition of Myxococcus xanthus, a fruiting myxobacterium. J Bacteriol 133, 763-768.

Browning, D. F., Whitworth, D. E. \& Hodgson, D. A. (2003). Lightinduced carotenogenesis in Myxococcus xanthus: functional characterization of the ECF sigma factor CarQ and antisigma factor CarR. Mol Microbiol 48, 237-251.

Campbell, E. A. \& Darst, S. A. (2000). The anti- $\sigma$ factor SpoIIAB forms a 2:1 complex with $\sigma^{\mathrm{F}}$, contacting multiple conserved regions of the $\sigma$ factor. J Mol Biol 300, 17-28.

Carrero-Lérida, J., Moraleda-Muñoz, A., Garcia-Hernández, R., Pérez, J. \& Muñoz-Dorado, J. (2005). PhoR1-PhoP1, a third twocomponent system of the family PhoRP from Myxococcus xanthus: role in development. J Bacteriol 187, 4976-4983.

Cayuela, M. L., Elías-Arnanz, M., Peñalver-Mellado, M., Padmanabhan, S. \& Murillo, F. J. (2003). The Stigmatella aurantiaca homolog of Myxococcus xanthus HMGA-type transcription factor CarD: insights into the functional modules of CarD and their distribution in bacteria. J Bacteriol 185, 3527-3537.

Cervantes, M. \& Murillo, F. J. (2002). Role for vitamin $B_{12}$ in light induction of gene expression in the bacterium Myxococcus xanthus. J Bacteriol 184, 2215-2224.

Cho, K. \& Zusman, D. R. (1999). AsgD, a new two-component regulator required for A-signalling and nutrient sensing during early development of Myxococcus xanthus. Mol Microbiol 34, 268-281.
Elías-Arnanz, M., Fontes, M. \& Padmanabhan, S. (2008). Carotenogenesis in Myxococcus xanthus: a complex regulatory network. In Myxobacteria: Multicellularity and Differentiation, pp. 211-225. Edited by D. E. Whitworth. Washington, DC: American Society for Microbiology.

Fontes, M., Ruiz-Vázquez, R. \& Murillo, F. J. (1993). Growth phase dependence of the activation of a bacterial gene for carotenoid synthesis by blue light. EMBO J 12, 1265-1275.

Fontes, M., Galbis-Martínez, L. \& Murillo, F. J. (2003). A novel regulatory gene for light-induced carotenoid synthesis in the bacterium Myxococcus xanthus. Mol Microbiol 47, 561-571.

Galbis-Martínez, M., Fontes, M. \& Murillo, F. J. (2004). The highmobility group A-type protein CarD of the bacterium Myxococcus xanthus as a transcription factor for several distinct vegetative genes. Genetics 167, 1585-1595.

Golemis, E. A. \& Serebriiskii, I. (2001). Recent developments in two hybrid technology. In Molecular Cloning: a Laboratory Manual, 3rd edn, pp. 18.16-18.47. Edited by J. Sambrook \& D. W. Russell. Cold Spring Harbor, NY: Cold Spring Harbor Laboratory.

Gorham, H. C., McGowan, S. J., Robson, P. \& Hodgson, D. A. (1996). Light-induced carotenogenesis in Myxococcus xanthus: light-dependent membrane sequestration of ECF sigma factor CarQ by anti-sigma factor CarR. Mol Microbiol 19, 171-186.

Gött, P. \& Boos, W. (1988). The transmembrane topology of the snglycerol-3-phosphate permease of Escherichia coli analysed by phoA and lacZ protein fusions. Mol Microbiol 2, 655-663.

Hanahan, D. (1983). Studies on transformation of Escherichia coli with plasmids. J Mol Biol 166, 557-580.

Ho, S. N., Hunt, H. D., Horton, R. M., Pullen, J. K. \& Pease, L. R. (1989). Site-directed mutagenesis by overlap extension using the polymerase chain reaction. Gene 77, 51-59.

Ho, M. S., Carniol, K. \& Losick, R. (2003). Evidence in support of a docking model for the release of the transcription factor $\sigma^{\mathrm{F}}$ from the antisigma factor SpoIIAB in Bacillus subtilis. J Biol Chem 278, 20898-20905.

Hodgson, D. A. (1993). Light-induced carotenogenesis in Myxococcus xanthus: genetic analysis of the carR region. Mol Microbiol 7, 471-488.

Karimova, G., Pidoux, J., Ullmann, A. \& Ladant, D. (1998). A bacterial two-hybrid system based on a reconstituted signal transduction pathway. Proc Natl Acad Sci U S A 95, 5752-5756.

Karimova, G., Ullmann, A. \& Ladant, D. (2000). A bacterial two-hybrid system that exploits a cAMP signaling cascade in Escherichia coli. Methods Enzymol 328, 59-73.

López-Rubio, J. J., Elias-Arnanz, M., Padmanabhan, S. \& Murillo, F. J. (2002). A repressor-antirepressor pair links two loci controlling lightinduced carotenogenesis in Myxococcus xanthus. J Biol Chem 277, 7262-7270.

López-Rubio, J. J., Padmanabhan, S., Lázaro, J. M., Salas, M., Murillo, F. J. \& Elías-Arnanz, M. (2004). Operator design and mechanism for CarA repressor-mediated down-regulation of the photoinducible carB operon in Myxococcus xanthus. J Biol Chem 279, 28945-28953.

Los, D. A. \& Murata, N. (1998). Structure and expression of fatty acid desaturases. Biochim Biophys Acta 1394, 3-15.

Martínez-Argudo, I., Ruiz-Vázquez, R. M. \& Murillo, F. J. (1998). The structure of an ECF- $\sigma$-dependent, light-inducible promoter from the bacterium Myxococcus xanthus. Mol Microbiol 30, 883-893.

Martínez-Laborda, A. \& Murillo, F. J. (1989). Genic and allelic interactions in the carotenogenic response of Myxococcus xanthus to blue light. Genetics 122, 481-490. 
McGowan, S. J., Gorham, H. C. \& Hodgson, D. A. (1993). Lightinduced carotenogenesis in Myxococcus xanthus: DNA sequence analysis of the carR region. Mol Microbiol 10, 713-735.

Mitchell, A. G. \& Martin, C. E. (1997). Fah1p, a Saccharomyces cerevisiae cytochrome b5 fusion protein, and its Arabidopsis thaliana homolog that lacks the cytochrome b5 domain both function in the alpha-hydroxylation of sphingolipid-associated very long chain fatty acids. J Biol Chem 272, 28281-28288.

Moraleda-Muñoz, A., Pérez, J., Fontes, M., Murillo, F. J. \& MuñozDorado, J. (2005). Copper-induction of carotenoid synthesis in the bacterium Myxococcus xanthus. Mol Microbiol 56, 1159-1168.

Moreno, A. J., Fontes, M. \& Murillo, F. J. (2001). ihfA gene of the bacterium Myxococcus xanthus and its role in activation of carotenoid genes by blue light. J Bacteriol 183, 557-569.

Navarro-Avilés, G., Jiménez, M. A., Pérez-Marín, M. C., González, C., Rico, M., Murillo, F. J., Elías-Arnanz, M. \& Padmanabhan, S. (2007). Structural basis for operator and antirepressor recognition by Myxococcus xanthus CarA repressor. Mol Microbiol 63, 980-994.

Nicolás, F. J., Ruiz-Vázquez, R. M. \& Murillo, F. J. (1994). A genetic link between light response and multicellular development in the bacterium Myxococcus xanthus. Genes Dev 8, 2375-2387.

Nicolás, F. J., Cayuela, M. L., Martínez-Argudo, I. M., Ruiz-Vázquez, R. M. \& Murillo, F. J. (1996). High mobility group I (Y)-like DNA domains on a bacterial transcription factor. Proc Natl Acad Sci U S A 93, 6881-6885.

Padmanabhan, S., Elias-Arnanz, M., Carpio, E., Aparicio, P. \& Murillo, F. J. (2001). Domain architecture of a high mobility group Atype bacterial transcriptional factor. J Biol Chem 276, 41566-41575.

Peñalver-Mellado, M., García-Heras, F., Padmanabhan, S., GarcíaMoreno, D., Murillo, F. J. \& Elías-Arnanz, M. (2006). Recruitment of a novel zinc-bound transcriptional factor by a bacterial HMGA-type protein is required for regulating multiple processes in Myxococcus xanthus. Mol Microbiol 61, 910-926.

Pérez-Marín, M. C., López-Rubio, J. J., Murillo, F. J., Elías-Arnanz, M. \& Padmanabhan, S. (2004). The N-terminus of Myxococcus xanthus CarA repressor is an autonomously folding domain that mediates physical and functional interactions with both operator DNA and antirepressor protein. J Biol Chem 279, 33093-33103.

Petit, F. \& Guespin-Michel, J. F. (1992). Production of an extracellular milk clotting activity during development in Myxococcus xanthus. J Bacteriol 174, 5136-5140.
Ruiz-Vázquez, R. M. \& Murillo, F. J. (1984). Abnormal motility and fruiting behavior of Myxococcus xanthus bacteriophage resistant strains induced by a clear plaque mutant of bacteriophage Mx8. J Bacteriol 160, 818-821.

Ruiz-Vázquez, R. M., Fontes, M. \& Murillo, F. J. (1993). Clustering and co-ordinated activation of carotenoid genes in Myxococcus xanthus by blue light. Mol Microbiol 10, 25-34.

Sambrook, J. \& Russell, D. W. (2001). Molecular Cloning: a Laboratory Manual, 3rd edn. Cold Spring Harbor, NY: Cold Spring Harbor Laboratory.

Shanklin, J., Whittle, E. \& Fox, B. G. (1994). Eight histidine residues are catalytically essential in a membrane-associated iron enzyme, stearoyl-CoA desaturase, and are conserved in alkane hydroxylase and xylene monooxygenase. Biochemistry 33, 12787-12794.

Shanklin, J., Achim, C., Schmidt, H., Fox, B. G. \& Munck, E. (1997). Mossbauer studies of alkane omega-hydroxylase: evidence for a diiron cluster in an integral-membrane enzyme. Proc Natl Acad Sci U S A 94, 2981-2986.

Stephens, K. \& Kaiser, D. (1987). Genetics of gliding motility in Myxococcus xanthus: molecular cloning of the $\mathrm{mgl}$ locus. Mol Gen Genet 207, 256-266.

Studier, F. W., Rosenberg, A. H., Dunn, J. J. \& Dubendorff, J. W. (1990). Use of T7 RNA polymerase to direct expression of cloned genes. Methods Enzymol 185, 60-89.

Thomson, T. M., Lozano, J. J., Loukili, N., Carrio, R., Serras, F., Cormand, B., Valeri, M., Diaz, V. M., Abril, J. \& other authors (2000). Fusion of the human gene for the polyubiquitination coeffector UEV1 with Kua, a newly identified gene. Genome Res 10, 1743-1756.

Whitworth, D. E. \& Hodgson, D. A. (2001). Light-induced carotenogenesis in Myxococcus xanthus: evidence that CarS acts as an antirepressor of CarA. Mol Microbiol 42, 809-819.

Yanisch-Perron, C., Vieira, J. \& Messing, J. (1985). Improved M13 phage cloning vectors and host strains: nucleotide sequences of the M13mp18 and pUC19 vectors. Gene 33, 103-119.

Yudkin, M. D. \& Clarkson, J. (2005). Differential gene expression in genetically identical sister cells: the initiation of sporulation in Bacillus subtilis. Mol Microbiol 56, 578-589.

Edited by: J.-H. Roe 